\title{
Theory of adsorption and surfactant effect of Sb on Ag (111)
}

\author{
Sabrina Oppo, \\ Fritz-Haber-Institut der Max-Planck-Gesellschaft, Faradayweg 4-6, D-14195 Berlin, Germany
}

(Submitted to Physical Review Letters on 13 July 1993)

\begin{abstract}
We present first-principles studies of the adsorption of $\mathrm{Sb}$ and $\mathrm{Ag}$ on clean and Sb-covered $\mathrm{Ag}$ (111). For Sb, the substitutional adsorption site is found to be greatly favored with respect to on-surface fcc sites and to subsurface sites, so that a segregating surface alloy layer is formed. Adsorbed silver adatoms are more strongly bound on clean $\mathrm{Ag}(111)$ than on Sb-covered Ag. We propose that the experimentally reported surfactant effect of $\mathrm{Sb}$ is due to $\mathrm{Sb}$ adsorbates reducing the $\mathrm{Ag}$ adatom mobility. This gives rise to a high density of $\mathrm{Ag}$ islands which coalesce into regular layers.

PACS numbers : 68.35.-p, 68.35.Bs, 68.35.Md
\end{abstract}

Typeset using REVTEX 
The goal of epitaxial crystal growth is to achieve atomically-flat and defect-free surfaces of specified crystallographic orientation, under the widest possible range of growth conditions. Significant efforts are devoted since many years to the growth of semiconductors. The epitaxial growth of metals on metal substrates has also attracted considerable interest (see for example Ref. [囬] and references therein).

Layer-by-layer, or two-dimensional (2D), growth is such that the epitaxial layer being currently deposited is completed before further layers begin to grow on top of it; this mode is also named Frank-van der Merwe. In the three-dimensional (3D) or cluster growth, many overlayers grow at the same time, none of them being completed, so that the surface exhibits 3D islands. For heteroepitaxy, depending on whether the 3D mode manifests itself immediately or only after the formation of a few $2 \mathrm{D}$ overlayers, the $3 \mathrm{D}$ growth is named either after Volmer and Weber, or Stransky and Krastanov.

With a view at extending the external conditions for the growth of high-quality 2D surfaces towards lower temperatures and higher deposition rates, use has been made recently of surface contaminants which purportedly act as surfactants. Although by definition [2] a surfactant should reduce the surface formation energy [3], there is at present no consensus as to the actual mechanisms of surfactant action, e.g. as to whether the contaminant affects the surface energy or the kinetics of growth [四]; the term surfactant is thus often used in the broader sense that it promotes $2 \mathrm{D}$ growth as opposed to $3 \mathrm{D}$ growth.

The surfactant technique, although still in its infancy, has been by now rather widely applied [3,4] in the field of semiconductors to help regular growth of heteroepitaxial strained layers. On the other hand, we are only aware of one report of surfactant-assisted growth of metals [5]. This is concerned with the homoepitaxy of the (111) surface of Ag. The growth mode was found to be drastically altered, from 3D to layer-by-layer 2D, by the one-time deposition of $\mathrm{Sb}$ at the beginning of the growth process, at coverages $\Theta$ between 0.05 and 0.2. Clean Ag (111) was observed to grow in a 3D fashion between 250 and $400 \mathrm{~K}$, as signaled by the exponential decrease of the reflected x-ray beam intensity which monitors the degree of coherence of the upper layers of the sample [5]. A crossover to step-flow 
growth (corresponding to constant reflected intensity) was observed above 450-500 K. In the presence of $\mathrm{Sb}$, an oscillatory behaviour of the reflected intensity was observed instead, which is a fingerprint of $2 \mathrm{D}$ growth. The layer-by-layer growth of Sb-precovered Ag (111) continues for a rather long time (typically equivalent to the growth of 25 monolayers or more), at a nominal Ag deposition rate of 0.02 monolayer per second, even at 280-300 K.

The actual growth mode of an ideal clean Ag surface is still unclear. Indeed, Ag would be expected to grow layer-by-layer even at rather low temperatures; 3D cluster growth might be initiated e.g. by nucleation at surface defects. This notwithstanding, Sb unambiguously promotes the $2 \mathrm{D}$ growth of $\mathrm{Ag}$, and it is therefore important to investigate the pertaining mechanism. With this aim, we have performed ab initio studies of the energetics of $\mathrm{Sb}$ and Ag adsorption on $\mathrm{Ag}$ (111). The calculations presented in this Letter are performed at $\mathrm{Sb}$ coverages down to $\Theta=1 / 4$. Among the considered adsorption geometries, the most stable one is the substitutional site, Sb being bound into a Ag surface vacancy. This site is considerably more favourable than the conventional on-surface fcc sites, and than subsurface sites. Thus, an Sb-Ag alloy layer forms in the surface layer. Dissolution of adsorbed Sb into bulk $\mathrm{Ag}$ is energetically disfavoured. When covered with $\mathrm{Ag}$, the substitutional surface alloy reforms as the topmost surface layer by segregation of Sb. Due to the need of forming surface vacancies, the formation of the substitutional surface alloy needs thermal activation; we predict that at the relevant temperature, at low enough coverages, disordering of the surface alloy should take place.

Calculations for Ag adsorbed on clean and substitutional Sb-covered Ag (111) give informations on the growth mode of Ag. The main result is that Ag is more bound on clean portions of the surface, while the vicinity of substitutional Sb centers is less favorable. This implies that the average barrier for $\mathrm{Ag}$ diffusion is increased, thus Sb reduces the surface mobility of Ag. This causes a high Ag island density, which reduces the probability of 3D growth. The segregation of Sb to the surface layer allows the process to continue.

The calculations were performed within density-functional theory [6] in the local density approximation (LDA), using the all-electron full-potential linear muffin-tin orbitals (LMTO) 
method [7]. We used a non-relativistic code, which gives a very good description of Ag (bulk equilibrium properties: $a_{0}^{\text {th }}=7.73 \mathrm{bohr}, B^{\text {th }}=1.10$ Mbar, zero-point energy not included, to be compared with the low-temperature experimental values $a_{0}^{\exp }=7.74 \mathrm{bohr}, B^{\exp }=1.01$ Mbar) and Sb, which is only slightly heavier. The (111) surface of fcc Ag, clean or with Sb coverages of $\Theta=1, \Theta=1 / 3$, and $\Theta=1 / 4$ (whereby the $1 \times 1,(\sqrt{3} \times \sqrt{3}) R 30^{\circ}$, and $2 \times 2$ cells were used respectively), was simulated by slabs of thickness ranging from 5 to 13 atomic layers, separated by 10 layers of vacuum. The supercells contained a number of atoms ranging from 7 to 30 . The $\mathbf{k}$-summation was done on a uniform mesh in the irreducible part of the surface Brillouin zone, encompassing 19 points for the $1 \times 1$ cell, 7 and 13 points for the clean and adsorbate-covered $\sqrt{3} \times \sqrt{3}$ cell, 5 and 9 points for the clean and adsorbatecovered $2 \times 2$ cell. The vertical position of the adsorbates was optimized. Substrate relaxation is neglected, but is expected to change the adsorption energies only marginally. Full details of this study will be presented elsewhere [8].

The binding energy for Sb on-surface fcc adsorption is

$$
E_{\mathrm{ad}}^{\mathrm{fcc}}=-\left(\frac{1}{2} E^{\mathrm{Sb} / \operatorname{Ag}(111)}-\frac{1}{2} E^{\mathrm{Ag}(111)}-E_{\mathrm{atom}}^{\mathrm{Sb}}\right)
$$

with $E^{\mathrm{Sb} / \mathrm{Ag}(111)}, E^{\mathrm{Ag}(111)}$, and $E_{\text {atom }}^{\mathrm{Sb}}$ being the total energies of the adsorbate-covered slab, of the clean $\mathrm{Ag}$ slab, and of the spin-polarized $\mathrm{Sb}$ free atom; the factor $1 / 2$ accounts for the facts that we adsorb on both slab sides. In the case of substitutional Sb adsorption, a slightly different definition applies:

$$
E_{\mathrm{ad}}^{\mathrm{sub}}=-\left[\left(\frac{1}{2} E^{\mathrm{Sb} / \mathrm{Ag}(111) \mathrm{sub}}+E_{\mathrm{bulk}}^{\mathrm{Ag}}\right)-\left(\frac{1}{2} E^{\mathrm{Ag}(111)}-E_{\mathrm{atom}}^{\mathrm{Sb}}\right)\right]
$$

where $E_{\mathrm{bulk}}^{\mathrm{Ag}}$ is the bulk total energy per atom of fcc Ag. The substitutional process implies in fact that a surface vacancy be created, and the kicked-out Ag atom migrates to a kink site at a surface step, thus gaining the cohesive energy [9]. While the formation of a surface vacancy costs energy, the subsequent binding of the adsorbate into the vacancy leads to a net energy gain. While at $\Theta=1$ we only have on-surface adsorption, substitutional adsorption with $\mathrm{Sb}$ adatoms being not nearest neighbours is possible for all $\Theta \leq 1 / 3$ (we did not consider the coverage $0.3<\Theta<1)$. 
The calculated adsorption energies for the substitutional, fcc on-surface, and sublayer

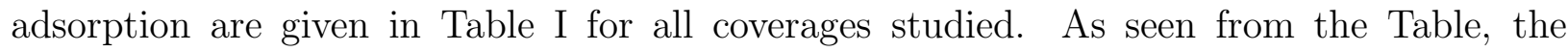
substitutional site is greatly favoured with respect to "normal" on-surface fcc adsorption, and also against sublayer adsorption. Sb is thus expected to be adsorbed in substitutional sites [10].

An obstacle to the establishment of a substitutional adsorbate superstructure is the energy barrier which may exist for vacancy formation. To estimate the barrier, we calculated the formation energy of a distant Frenkel pair [9], consisting of an isolated Ag adatom on Ag (111) plus a vacancy. The results are summarized in Table [1]. The resulting maximum barrier of about $1.5 \mathrm{eV}$ corresponds to an activation temperature of about $500 \mathrm{~K}$. At that temperature the surface mobility of $\mathrm{Ag}$ atoms on $\mathrm{Ag}$ (111) is very high, so that migration of the atom released from the vacancy to a kink site is easily achieved. We note that, if dissipated locally, the adsorption energy of $\mathrm{Sb}$ in the fcc site would be more than enough to create a surface vacancy.

As a check as to whether $\mathrm{Sb}$ might be incorporated into the bulk of $\mathrm{Ag}$, we calculated the adsorption energy for $\mathrm{Sb}$ in a sublayer site, i.e. below one overlayer of Ag. As seen from Table $\llbracket$, this site is strongly disfavored with respect to Sb sitting in a substitutional site in the surface layer. For Sb below two Ag overlayers, the adsorption energy decreases further. We conclude that $\mathrm{Sb}$ segregates to the surface and is not incorporated into Ag. Indeed, the segregation of $\mathrm{Sb}$ (and the ensuing reduction of surface energy) in transition and noble metals and alloys has been known for some time in metallurgy [11]. In the present case, segregation is essentially due to the size difference of $\mathrm{Ag}$ and $\mathrm{Sb}$, as has been checked by additional calculations at a $5 \%$ increased lattice constant. For $\Theta=1 / 3$, this gives that the difference of substitutional and sublayer adsorption energies drops from $1.1 \mathrm{eV} /$ atom to about $0.65 \mathrm{eV} /$ atom. $\mathrm{Sb}$ is just about the right size to fit into a surface vacancy, but it is somewhat too large for a bulk vacancy. Since $\mathrm{Sb}$ is confined into the surface layer, an $\mathrm{Sb}-\mathrm{Ag}$ alloy layer will form at the (111) surface of Ag upon submonolayer Sb deposition. It is worth noticing that the substitutional configuration on the fcc (111) surface has a first-neighbour 
geometry very close to that of $\mathrm{SbAg}_{3}$, the only stable ordered $\mathrm{Sb}-\mathrm{Ag}$ compound known, having a tetragonally-distorted fcc structure [12].

The substitutional $\mathrm{Sb}$ adsorbate sits in the surface vacancy in a position very close to the ideal fcc location of the substituted Ag atom, with an outward relaxation of only 5 to $8 \%$ of the interlayer spacing, ie. about 0.25-0.35 $\AA$. Due to the effective in-plane screening thus provided by the surrounding substrate atoms, the substitutional Sb adatoms interact only weakly with each other; the adsorption energy for the substitutional site does not change much at low coverage if the local environment for substitutional Sb is conserved (see Table 四). If we assume the $\Theta=1 / 4$ adsorption energy to be the low coverage limit value, and the coverage to be low enough, the entropic contribution to the free energy can overcome the internal energy difference at relatively low temperatures. At a coverage of $\Theta \simeq 0.1$, the annealing temperature (say, $600 \mathrm{~K}$ ) is sufficient to cause disordering with respect to the $\sqrt{3} \times \sqrt{3}$ arrangement. If the substitutional adsorption is activated by annealing, we therefore expect that the substitutional surface alloy thus obtained will be disordered.

To clarify the effects of Sb adsorption on the growth mode of Ag, we studied Ag adsorption on clean and Sb-covered $\mathrm{Ag}$ (111). We used the $2 \times 2$ cell for these studies, both because neighboring adsorbates are reasonably decoupled from each other, and because Ag can be adsorbed on the substitutional Sb-covered surface either as a nearest neighbour to Sb, or not. We call these two sites "near" and "far". The adsorption energies are summarized in Table [II]. The main result is that Ag has a higher adsorption energy on clean Ag than at both of the sites on Sb-covered Ag. Among the latter sites, the "near" site is marginally disfavored, and it would be probably more so if Sb had been allowed to relax outwards (see Table (1).

It is thus energetically preferrable for $\mathrm{Ag}$ to sit on clean portions of the surface, while the vicinity of substitutional Sb centers is unfavourable. This could be called long-range "site" blocking, as the interaction giving rise to it is apparently long-ranged. We put "site" in quotes because the adsorbate potential energy is expected to change gradually as the adsorbate approaches the Sb centers, so that the average diffusion barrier for Ag increases 
already at some distance from, and not only at, the Sb centers. Diffusion barriers for Ag on $\mathrm{Ag}$ (111) are smaller than $0.1 \mathrm{eV}$ : near an Sb center, they increase significantly, namely to about $0.4-0.5 \mathrm{eV}$. As a consequence, adsorbed $\mathrm{Sb}$ in the substitutional configuration reduces the surface mobility of Ag. The presence of substitutional Sb should therefore favor the growth of small-sized Ag islands. If the island density is high, one expects that they coalesce into a single layer before overgrowth on the islands can occur, as it is generally believed that small islands have lower energy barriers at descending steps. As deposited Ag covers the Ag:Sb surface alloy layer, Sb atoms find themselves in the disfavoured sublayer configuration, and will thus tend to segregate to the new surface layer. The alloy surface layer is thus reestablished, and the process can start again.

Recent STM experiments [13] on this system have indeed shown that on annealed Sbcovered surfaces, Sb induces a high density of small Ag islands on the surface, and that it efficiently segregates upon deposition of Ag. Another observation is that upon annealing at about $550 \mathrm{~K}$, Sb is adsorbed substitutionally and, at very low coverages, it forms a disordered 2D array, in agreement with our prediction. For the unannealed surface, the on-surface fcc site is occupied at room temperature; this agrees with our estimate of the activation of substitutional adsorption. At very low coverage, $\mathrm{Sb}$ is observed to form islands. Our largest calculated adsorption energy for on-surface adsorption is that of the $2 \times 2$ superstructure; we cannot exclude however that the adsorption energy may increase further in the extreme low-coverage limit, which is computationally very demanding and has not been addressed here.

In summary, we presented ab initio calculations of $\mathrm{Sb}$ and $\mathrm{Ag}$ adsorption on clean and Sbcovered Ag (111). For Sb, the substitutional adsorption site is energetically highly favoured with respect to "normal" on-surface sites; in addition, subsurface positions are also strongly disfavoured. Sb is thus effectively confined into the surface and forms a segregating surface alloy. This alloy should disorder, at low coverages, for typical annealing temperatures. As to $\mathrm{Ag}$, we find it to be sizably more bound on clean $\mathrm{Ag}(111)$ than on substitutional Sb-covered Ag: this indicates that Sb produces a site blocking, or more precisely, a significant increase 
of the diffusion barrier for Ag adatoms approaching the Sb centers. Based on these results, we offered an explanation of the recently observed Sb-induced layer-by-layer homoepitaxial growth of $\mathrm{Ag}$ (111): substitutionally-adsorbed Sb induces, by mobility reduction, a high density of small-sized Ag islands which coalesce into a regular 2D layer; as Ag covers the surface, Sb segregates to the newly formed layer, thus reestablishing the alloy layer at the surface, and the process starts again. Most of our results seem to be confirmed by recent STM experiments [13].

We thank R. Stumpf for helpful discussions, and J. Vrijmoet for communicating his results prior to publication. This work was partly supported by the Deutsche Forschungsgemeinschaft within Sonderforschungsbereich 1421. 


\section{REFERENCES}

* Present address: Dipartimento di Scienze Fisiche, Università di Cagliari, via Ospedale 72, I-09124 Cagliari, Italy.

[1] F. J. A. den Broeder, D. Kuiper, A. P. van den Mosselaer, and W. Hoving, Phys. Rev. Lett. 60, 2769 (1988); W. F. Egelhoff and I. Jacob,ibid. 62, 921 (1989); M. Bott, T. Michely, and G. Comsa, Surf. Sci, 272, 161 (1992).

[2] Oxford Dictionary of Physics (Oxford UP, Oxford 1988).

[3] D. J. Eaglesham, F. C. Unterwald, and D. C. Jacobson, Phys. Rev. Lett. 70, 966 (1993), and references therein.

[4] C. W. Snyder and B. G. Orr, Phys. Rev. Lett. 70, 1030 (1993); N. Grandjean and J. Massies, ibid., 1031, and references therein.

[5] H. A. van der Vegt, H. M. van Pinxteren, M. Lohmeier, E. Vlieg, and J. M. C. Thornton, Phys. Rev. Lett. 68, 3335 (1992).

[6] See e.g. R. Dreizler and E. Gross, Density functional theory, (Springer, Berlin, 1990). The exchange-correlation energy is by D. M. Ceperley and B. J. Alder, Phys. Rev. Lett. 45, 566 (1980), as parametrized by S. Wosko, L. Wilk, and M. Nusair, Can. J. Phys. 58, $1200(1980)$.

[7] M. Methfessel, Phys. Rev. B 38, 1537 (1988); M. Methfessel, C. O. Rodriguez, and O. K. Andersen, ibid. 40, 2009 (1989). For surface studies, see M. Methfessel, D. Hennig, and M. Scheffler, Phys. Rev. B 46, 4816 (1992).

[8] S. Oppo, V. Fiorentini, and M. Scheffler, to be published.

[9] J. Neugebauer and M. Scheffler, Phys. Rev 46, 16067 (1992); A. Schmalz, S. Aminpirooz, L. Becker, J. Haase, J. Neugebauer, M. Scheffler, D. R. Batchelor, D. L. Adams, and E. Bøgh, Phys. Rev. Lett. 67, 2163 (1991). 
[10] The results may be reformulated in terms of surface energy reduction caused by adsorption. This requires the definition of a chemical potential for the atoms to be adsorbed. Here we adopt the adsorption energy terminology.

[11] See e.g. M. Jenko, F. Vodopivec, and B. Praček, Appl. Surf. Sci. 70/71, 118 (1993) and references therein; M. Hondros and A. Mc Lean, in Surface Phenomena of Metals, Soc. Chem. Ind. Monograph Nr. 28 (Soc. Chem. Ind., London 1969), p. 39.

[12] P. Villars, K. Matthis, and F. Hulliger, in The structure of binary compounds, F. R. De Boer and D. G. Pettifor eds., p.1 (Elsevier, Amsterdam, 1989).

[13] J. Vrijmoeth, private communication. 


\section{TABLES}

\begin{tabular}{l|ccc}
\hline \hline \multicolumn{1}{c}{} & $\sqrt{3} \times \sqrt{3}$ & $2 \times 2$ & $1 \times 1$ \\
\hline $\mathrm{E}_{\text {ad }}^{\text {sub }}$ & 4.49 & 4.37 & - \\
relaxation & $+5 \%$ & $+8 \%$ & - \\
\hline $\mathrm{E}_{\text {ad }}^{\mathrm{fcc}}$ & 3.26 & 3.34 & 3.22 \\
relaxation & $-11 \%$ & $-5 \%$ & $+6 \%$ \\
\hline $\mathrm{E}_{\text {ad }}^{\text {sublayer }}$ & 3.41 & 3.45 & 2.71 \\
\hline \hline
\end{tabular}

TABLE I. Adsorption energies (in eV/atom) of $\mathrm{Sb}$ on $\mathrm{Ag}$ (111) for the adsorption sites and coverages studied here. Vertical adsorbate relaxations compared to ideal silver fcc position (in percentage of interlayer spacing) are also given.

\begin{tabular}{|c|c|c|}
\hline & $\sqrt{3} \times \sqrt{3}$ & $2 \times 2$ \\
\hline $\mathrm{E}_{\mathrm{f}}^{\mathrm{vac}}$ & 0.69 & 0.66 \\
\hline $\mathrm{E}_{\mathrm{f}}^{\mathrm{Fp}}$ & 1.46 & 1.43 \\
\hline
\end{tabular}

TABLE II. Vacancy and Frenkel pair formation energies (eV)

\begin{tabular}{|c|c|c|c|}
\hline & clean & far & near \\
\hline$E_{\mathrm{ads}}^{\mathrm{Ag}}$ & 2.41 & 2.02 & 1.99 \\
\hline relaxation & $-9 \%$ & $-9 \%$ & $-5 \%$ \\
\hline
\end{tabular}

TABLE III. Adsorption energy (eV/atom) and relaxation compared to the ideal Ag position

(in percentage of ideal interlayer spacing) for Ag on clean and Sb-covered Ag (111). Clean: Ag on $\operatorname{Ag}(111)$; far: $\mathrm{Ag}$ on $\mathrm{Sb}: \mathrm{Ag}(111)$, "far" site; near: $\mathrm{Ag}$ on $\mathrm{Sb}: \mathrm{Ag}(111)$, "near" site. 